\title{
Combinatorial Formulation of Ising Model Revisited
}

\author{
G.A.T.F. da Costa* and A. L. Maciel ${ }^{\dagger}$ \\ Departamento de Matemática \\ Universidade Federal de Santa Catarina \\ 88040-900, Florianópolis, SC, Brasil
}

Recebido em 15 de julho, 2002. Aceito em 12 de novembro, 2002.

\begin{abstract}
In 1952, Kac and Ward developed a combinatorial formulation for the two-dimensional Ising model which is another method of obtaining Onsager's famous formula for the free energy per site in the termodynamic limit of the model. Feynman gave an important contribution to this formulation conjecturing a crucial mathematical relation which completed Kac and Ward ideas. In this paper, the method of Kac, Ward and Feynman for the free field Ising model in two dimensions is reviewed in a self-contained way and Onsager's formula is computed.
\end{abstract}

Em 1952, Kac e Ward desenvolveram uma formulação combinatorial do modelo de Ising em duas dimensões que é um outro método para se obter a famosa fórmula de Onsager para a energia livre por sítio no limite termodinâmico do modelo. Feynman fez importante contribuição a esta formulação conjecturando uma relação matemática crucial que completou as idéias de Kac e Ward. Neste trabalho, o método de Kac, Ward e Feynman para o modelo de Ising em duas dimensões sem campo é revisada e a fórmula de Onsager é calculada.

\section{Introduction}

The aim of statistical physics is to understand the macroscopic behaviour of a system formed by a very large number of particles from information about how they interact with each other. One way in which one can gain insight into this problem and thus about complex systems is by constructing idealized models which hopefully will exhibit some of the interesting features of real systems like phase transitions. Perhaps the most studied of these idealized models is the Ising model so called in honor to his first investigator, Ernst Ising (1900-1998).

The model was originally proposed as a simple model of ferromagnetism. In ref. [1] Ising investigated the model in one dimension and computed exactly its partition function. In 1944, Onsager [2] considered the free field model in two dimensions and succeded to compute the partition function exactly. His method became known as the algebraic formulation of the model. In 1952, Kac and Ward [3] developed a quite different method of obtaining Onsager results known as the combinatorial formulation of the Ising model. Feynman developed the method farther and conjectured an identity relating functions defined on graphs and functions defined on paths on a square lattice $[4,7]$. This identity is a crucial element in the combinatorial formulation of Kac, Ward and Feynman of the Ising model. The identity was later formally proved by Sherman [4-6], followed later

\footnotetext{
*e-mail: gatcosta@mtm.ufsc.br

${ }^{\dagger}$ Suported by a PIBIC/CNPQ - BIP/UFSC fellowship
}

on by another proof by Burgoyne [7]. A somewhat similar treatment to the combinatorial formulation of Kac, Ward and Feynman can be found in refs. [12-14]. An important variant of the combinatorial formulation using the so called Pffafians was developed by Green and Hurst [10].

The bibliography on the Ising model is vast and to give a full list of references is virtually impossible. A nice introduction to the model though is the paper by B. Cipra given in ref. [17]. Old surveys but still useful on the distinct formulations of the Ising model in two dimensions and its history can be found in refs. [10-11, 15-16] together with full lists of original references.

The objective of the present paper is to review in a selfcontained way the calculation of the Onsager's formula for the two dimensional free field Ising model in the combinatorial formulation of Kac, Ward and Feynman. Our presentation follows chapter V, section V.4, of Feynman's book [9] and the paper by Burgoyne [7] although we have tried to be more careful with the mathematics involved than these references are.

The paper is organized as follows. In section II, the Ising model is defined. In section III and through its various subsections the combinatorial formulation of Kac, Ward and Feynman of the partition function is given. In section IV, Onsager's formula for the free energy per site in the thermodynamic limit is computed. 


\section{Definition of the model}

The model is defined on a finite planar square lattice $\Lambda$ which mimic a regular arranjement of atoms in two dimensions. Suppose the lattice is embedded in the plane with sites having coordinates in $\mathbf{Z} \times \mathbf{Z}$. To each site $i$ of $\Lambda$ it is assigned two possible states also called "spins" and denoted by $\sigma_{i}$, where $\sigma_{i}=+1$ or $\sigma_{i}=-1$. The interaction energy between two particles located at the $i$-th and $j$-th sites and in the states $\sigma_{i}$ and $\sigma_{j}$, respectively, is postulated to be

$$
E_{i j}= \begin{cases}-J \sigma_{i} \sigma_{j} & \text { if } i, j \text { are n.n. } \\ 0 & \text { otherwise }\end{cases}
$$

where "n.n" stands for nearest neighbors, hence, in the Ising model it is assumed that the energy depends only on short range interactions. The energy is $-J$ if the nearest neighbors are in the same state and $+J$ if the states are distinct. The constant $J$ which can be positive or negative is a parameter for the model.

Suppose $\Lambda$ has $N^{2}$ sites. Then, there are $2^{N^{2}}$ distinct configurations of the spins and, therefore, $2^{N^{2}}$ configurations $\sigma=\left(\sigma_{1}, \ldots, \sigma_{N^{2}}\right)$ of the system. Call $S=\{\sigma\}$ the set of possible configurations of the system. The energy of each configuration $\sigma \in S$ is given by

$$
E_{\sigma}=-J \sum_{n . n . \in \sigma} \sigma_{i} \sigma_{j}
$$

Suppose as well the system is at equilibrium temperature given by $T$. According to statistical mechanics, the probability $p_{\sigma}$ to find the system in the configuration $\sigma$ is

$$
p_{\sigma}=\frac{1}{Z(\beta)} e^{-\beta E_{\sigma}}
$$

where $\beta=\frac{1}{k_{B} T}, k_{B}$ is Boltzmann constant, and

$$
Z(\beta)=\sum_{\sigma} e^{-\beta E_{\sigma}} .
$$

is the so called partition function of the model. This simple looking function is simple to compute exactly only in one dimension, difficult but possible to compute exactly in dimension two. In three dimensions nobody knows how to do it.

The exact knowledge of $Z(\beta)$ allows one to obtain information about the global behaviour of the system. Important quantities that are relevant to understand the physics of the system are all defined in terms of $\ln Z$ or its derivatives. For instance, the free energy per lattice site $f$ in the thermodynamic limit is defined as

$$
f=-k_{B} T \lim _{N \rightarrow \infty} \frac{\log Z}{N^{2}} .
$$

A basic problem is to find a closed form, analytic expression for $f$. Phase transitions will appear as singularities in $f$ or in one of its derivatives.

\section{The combinatorial formulation}

In the combinatorial formulation the partition function is expressed as a sum over special subsets of the lattice $\Lambda$ called admissible graphs. Next, using a relation first conjectured by $\mathrm{R}$. Feynman the resulting expression is converted into a product over paths. The final step towards the Onsager's formula to be accomplished in section IV consists in deriving an integral representation for this product.

\section{III.1 The partition function as a sum over graphs}

Let's rewrite the partition function (2.4) as

$$
Z_{N}(K)=\sum_{\sigma_{1}= \pm 1} \cdots \sum_{\sigma_{N}= \pm 1} \prod_{n . n .} e^{K \sigma_{i} \sigma_{j}}
$$

with $K=+\frac{J}{k_{B} T}$. Noting that $\sigma_{i} \sigma_{j}= \pm 1$, it follows that

$$
e^{K \sigma_{i} \sigma_{j}}=e^{ \pm K}=\cosh K \pm \sinh K
$$

and

$$
\prod_{n . n .} e^{K \sigma_{i} \sigma_{j}}=\left(1-u^{2}\right)^{-\frac{x}{2}} \prod_{n . n .}\left(1+\sigma_{i} \sigma_{j} u\right)
$$

where $u=\tanh K$ and $x=2 N(N-1)$ is the number of bonds in $\Lambda$. Notice that $|u|<1$, for any $K$.

Definition 3.1. An admissible graph is a connected or disconnected subset of $\Lambda$ whose sites have even valence.

Definition 3.2. Given an admissible graph $G$, define

$$
I_{G}(u)=\prod_{i \in G} u=u^{L}
$$

where the product is over the bonds $i$ of $G$.

Theorem 3.1. Call $\mathcal{A}$ the set of all admissible graphs $G$ of $\Lambda$. Then,

$$
Z_{N}(u)=2^{N^{2}}\left(1-u^{2}\right)^{-N(N-1)}\left(1+\sum_{G \in \mathcal{A}} I_{G}(u)\right)
$$

Proof: To each pair $i, j$ of nearest neighbors of $\Lambda$ there correspond a term $u \sigma_{i} \sigma_{j}$ and a bond. Since the number of pairs $i, j$ of n.n. coincide with the number $x=2 N(N-1)$ of bonds of $\Lambda$ the product on the RHS of (3.3) is a polinomial of degree $x$, that is,

$$
\prod_{n . n .}\left(1+u \sigma_{i} \sigma_{j}\right)=1+\sum_{p=1}^{x} u^{p} \sum_{n . n .}\left(\sigma_{i_{1}} \sigma_{i_{2}}\right) \cdots\left(\sigma_{i_{2 p-1}} \sigma_{i_{2 p}}\right)
$$

The second summation is over all possible products of $p$ pairs $\left(\sigma_{i} \sigma_{j}\right)$ of n.n. of $\Lambda$ where a pair is not to occur twice in the same product. To each pair $\left(\sigma_{i} \sigma_{j}\right)$ there is associated a bond connecting the neighbors $i$ and $j$ so to each product of $p$ pairs correspond a graph (connected or disconnected). So, 
the second summation is over all graphs with $p$ bonds. The graphs may have sites with valence $1,2,3$ or 4 . The summations over the spins $\sigma_{i}$ 's eliminate graphs having sites with odd valence because $\sum \sigma_{i}=0$ and $\sum \sigma_{i}^{3}=0$. The graphs left are those whose sites have valence 2 or 4 , thus admissible. If $V_{G}$ is the number of sites in a admissible graph $G$ then there is a factor $2^{V_{G}}$ associated to it because each site of $G$ contributes a factor 2 coming from $\sum \sigma_{i}^{2}=2$ and $\sum \sigma_{i}^{4}=2$. The sum over $\sigma$ includes all the $\sigma_{i}$ and not only those with sites $i$ in $G$. The summation over the sites not in $G$ will give a factor $2^{V-V_{G}}$ where $V=N^{2}$ is the number of sites in the lattice, hence, in the end one gets the factor $2^{V}$.

\section{III.2 The partition function as a product over paths}

Let's orient and number the bonds of $\Lambda$ with distinct positive integers $i$ and call $\Lambda$ with this indexation a colored lattice.

Definition 3.3. A path $p$ over $\Lambda$ is an ordered sequence of bonds each starting at the site where the previous one ended. The last bond ending at the site from which the first one started. Thus, $p$ is closed. The path is subjected to the constraint that it never goes backwards over the previous bond. A path $p$ is given by a word, that is, an ordered sequence of symbols $D_{i}$ where $i$ distiguishes the bonds of $\Lambda$. A path $p$ is then of the form

$$
p=D_{j_{1}}^{e_{1}} D_{j_{2}}^{e_{2}} \ldots D_{j_{l}}^{e_{l}}
$$

for some $l$ and where $e_{i}=+1(-1)$ if the path traverses bond $j_{i}$ following the direction (opposite direction) assigned to it. Because a path is closed it is defined to within its circular order so that

$$
D_{j_{1}}^{e_{1}} D_{j_{2}}^{e_{2}} \ldots D_{j_{l}}^{e_{l}} \equiv D_{j_{2}}^{e_{2}} \ldots D_{j_{l}}^{e_{l}} D_{j_{1}}^{e_{1}} \equiv \ldots \equiv D_{j_{l}}^{e_{l}} D_{j_{1}}^{e_{1}} \ldots D_{j_{l-1}}^{e_{l-1}}
$$

The inversion $p^{-1}$ of $p$ is given by

$$
D_{j_{l}}^{-e_{l}} D_{j_{l-1}}^{-e_{l-1}} \ldots D_{j_{1}}^{-e_{1}}
$$

We take $p$ and $p^{-1}$ to be equivalent. Given $p$, denote by $[p]$ the set of all paths equivalent to $p$, that is, its circular permutations and their inversions.

Definition 3.4. A periodic path is one which has the word representation

$$
\left(D_{j_{1}}^{e_{1}} \ldots D_{j_{l}}^{e_{l}}\right)^{w}
$$

for some $l$ and $w \geq 2$ and where the subword in between brackets is nonperiodic.

Definition 3.5. A path $p$ has assigned to it a sign given by

$$
s(p)=(-1)^{1+t}
$$

where $t$ is the number of $2 \pi$-angles turned by a tangent vector while traversing $p$. A positive (negative) angle is assigned to a counterclockwise (clockwise) rotation.

Example 1. See Figure 1a). A tangent vector starting at point $e$ and traversing the path shown in Figure 1a) turns once a total angle given by $4 . \frac{\pi}{2}=2 \pi$ after its return to $e$ so in this case $t=1$ and $s(p)=+1$. For the path in Figure $1 \mathrm{~b})$, the total angle turned is $3 \frac{\pi}{2}-3 \frac{\pi}{2}=0$ so $t=0$ and $s(p)=-1$.

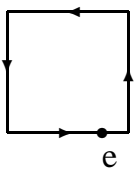

(a)

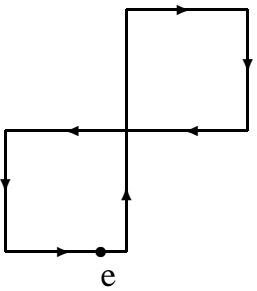

(b)
Figure 1: Examples of paths with (a) $s(p)=+1$

$$
\text { and (b) } s(p)=-1 \text {. }
$$

Remark. In section IV, instead of assigning an angle $\pm \pi / 2$ to a turn we will count the contribution to the sign by assigning $\alpha=e^{i \pi / 4}$ and $\bar{\alpha}=e^{-i \pi / 4}$ to each counterclockwise and clockwise turn, respectively, and then in the end multiplying the result by -1 . In the example above, one gets in this manner $\left(e^{i \pi / 4}\right)^{4}=-1$ and $\left(e^{i \pi / 4}\right)^{3} \cdot\left(e^{-i \pi / 4}\right)^{3}=+1$. Multiplying both results by -1 , one recovers the correct sign for each path.

The sign of periodic paths. Suppose the sign of the nonperiodic path in between brackets in (3.10) is $(-1)^{1+t_{s}}$. Then, the sign of the periodic path with period $w$ is $(-1)^{1+w t_{s}}$. Hence, the sign of a periodic path is -1 if its period is an even number and the sign equals the sign of the nonperiodic subpath if the period $w$ is an odd number.

Definition 3.6. To each path $p$ it is assigned the function $I_{p}(u)$ given by

$$
I_{p}(u)=u^{l}
$$

where $l=m_{1}+\ldots+m_{k}$, for some $k$, is the length of $p, m_{i}$ being the number of times bond $i$ is covered by $p$, and the function $W_{p}(u)$, "the amplitude of $p$ ", defined as follows:

$$
W_{p}(u)=s(p) I_{p}(u)
$$

Theorem 3.2. The functions $I_{G}(u)$ and $W_{p}(u),|u|<1$, defined above satisfy the following relation:

$$
1+\sum_{G \in \mathcal{A}} I_{G}(u)=\prod_{[p]}\left[1+W_{p}(u)\right]
$$

The product is over all inequivalent classes $[p]$ of closed nonperiodic paths. The summation is over all admissible graphs of the finite $N \times N$ planar square lattice $\Lambda$.

Relation (3.14) is a simpler version suitable for the Ising model of a more general relation investigated by Sherman and Burgoyne in refs. [4-7]. The difference is that they assign to the bonds $i$ of the lattice distinct parameters $d_{i}$, hence, in this case the functions $I_{G}$ and $W$ are given in terms of these parameters. In the Ising model context under consideration these are all equal to $u$ and $|u|<1$. 
According to references $[4,7,10,11]$, relation (3.14) first appeared as a conjecture in lecture notes by Feynman ( ref. [9], published only in 1972 and already mentioning ref. [4]). The first proof of it was achieved by Sherman in refs. [4,6] followed by another one later on by Burgoyne in ref. [7]. The simplest nontrivial case of the general relation is investigated in ref. [8].

Below Burgoyne's proof is essencially reproduced for the case $|u|<1$.

Proof: Expand the product over the distinct classes of nonperiodic paths $[p]$ as 1 (one) plus an infinite sum of terms of the form

$$
W_{p_{1}}(u) W_{p_{2}}(u) \ldots W_{p_{k}}(u)=s \prod_{i} u^{r_{i}}
$$

for some $k$ where $p_{1}, \ldots, p_{k}$ is a set of nonperiodic paths over $\Lambda$. The product on the r.h.s of (3.15) is over the bonds $i$ traversed by $p_{1}, p_{2}, \ldots p_{k}$, and $r_{i}$ says how many times. If $p_{1}, p_{2}, \ldots p_{k}$ traverse bond $i$, say, $m_{1}(i), \ldots, m_{k}(i)$ times, $m_{j} \geq 0$, respectively, then $r_{i}=\sum_{i=1}^{k} m_{j}(i)$. The sign $s$ is the product of the signs of $p_{1}, p_{2}, \ldots, p_{k}$.

Let's prove, first, that those terms having $r_{i}=1, \forall i$, add up to $\sum I_{G}(u)$. Consider one of these terms with associated paths $p_{1}, p_{2}, \ldots, p_{k}$. Each bond in the set of bonds traversed by $p_{1}, p_{2}, \ldots, p_{k}$ is traversed only once by one of these paths. Thus, the only possible intersection if any between any two of these paths in this case can occur only at a site of valence 4 and they cross each other like in Fig. (2.a)

. Otherwise, they are disjoint. Thus, the set of bonds traversed by paths $p_{1}, \ldots, p_{k}$ constitute a graph whose vertices have valence 2 or 4 . This is an admissible graph. Therefore, to each term of the form of (3.15) with $r_{i}=1, \forall i$, one can associate an admissible graph. This graph can be disconnected. This happen if the set of paths can be split into subsets completely disjoint which generate admissible graphs without any bonds and vertices in common.

Now, given an admissible graph $G$ one can in general associate more than one term of the form of (3.15) with $r_{i}=1$, each associated with a distinct set of paths. Let's see how this follows. The sites of an admissible graph have valence 2 or 4 . When a path strikes a site of valence 4 it has only 3 possible directions to follow. See Figures $2 a, 2 b$, 2c. (The case in Fig. $2 d$ is forbidden.) Then, any two terms associated to a given admissible graph $G$ will differ only in the types of crossings at the sites of $G$. Since there are 3 types of crossing per valence 4 site, the number of possible terms associated to $G$ is $3^{V}$ where $V$ is the number of sites of $G$ with valence 4 .

A term has a sign which comes out from the contribution of the signs of the paths associated to that term. Let's see how the sign of a term comes out. A term with $t_{1}$ crossings of type 1 (Fig. 2a.) has a sign which can be expressed as $(-1)^{t_{1}}$ where $t_{1}$ includes selfcrossings of single paths plus crossings between different paths. Indeed, since distinct closed paths always intersect in a even number of crossings then $(-1)^{t_{1}}$ will give the correct sign of the term which is the product of the signs of the individual paths. Let's asso- ciate to the crossings of type $j=2,3$ the sign $(+1)^{t_{2}}(+1)^{t_{3}}$ so that a term with $t_{j}$ crossings of type $j=1,2,3$ has a sign given by $(-1)^{t_{1}}(+1)^{t_{2}}(+1)^{t_{3}}$.

There are $V$ ! ways of distributing $V=t_{1}+t_{2}+t_{3}$ crossings among the sites of $G$ but since there are $t_{j}$ crossings of the type $j, j=1,2,3$, one has to divide $V$ ! by $t_{1} ! t_{2} ! t_{3}$ ! so that the number of distinct terms with $t_{j}$ crossings of type $j$ is

$$
\frac{V !}{t_{1} ! t_{2} ! t_{3} !}
$$

These terms have the same factor $I(G)=u^{L}$ where $L$ is the number of bonds of $G$. Summing all these terms arising from a given $G$ and summing over all admissible graphs $G$ of $\Lambda$ the result is

$$
\left(\sum_{G} \sum_{\{t\}} \frac{V !}{t_{1} ! t_{2} ! t_{3} !}(-1)^{t_{1}}(+1)^{t_{2}}(+1)^{t_{3}}\right) I(G)
$$

where $\sum_{t}$ means summation over all $t_{1}, t_{2}, t_{3}$ such that $t_{1}+t_{2}+t_{3}=V$. Using the multinomial theorem the summation over $\{t\}$ gives $(-1+1+1)^{V}$ and one gets the result $\sum_{G} I(G)$.

If $G$ is disconnected with $l$ components $G_{i}, i=$ $1,2, \ldots, l$, each of them with $t_{j}, j=1,2, \ldots, l$, sites of valence 4 and $\sum t_{j}=V$, then applying the previous argument to each component will give $I\left(G_{1}\right) I\left(G_{2}\right) \ldots I\left(G_{l}\right)=I(G)$.

In view of the above result, the theorem could be equivalently stated by saying that the sum of terms with $r_{i}>1$ for at least one of the $i$ converges to zero. Let's prove this.

Let $\mathcal{G}$ be the set of all colored connected or disconnected subgraphs $g$ of the colored lattice without valence 1 sites and such that if $g$ is connected then $g$ is not a poligon, that is, a graph having valence 2 sites only. A disconnected graph is allowed to have some but not all of the components as poligons. The reason for excluding graphs which are poligons or having all components which are is that closed paths with repeated bonds over them are necessarily periodic and these are forbidden. The coloring of $g$ is that inherited from the colored lattice.

Given $g \in \mathcal{G}$, call $i_{1}, \ldots, i_{l(g)}$ the bonds of $g$. A term $w_{g}$ associated to $g$ is of the form

$$
w_{g}=W_{p_{1}} W_{p_{2}} \ldots W_{p_{k}}=\left(\operatorname{sign} w_{g}\right)\left|w_{g}\right|
$$

for some $k$ and set of paths $p_{1}, \ldots, p_{k}$, which traverse the bonds of $g$ only, where

$$
\left|w_{g}\right|=\prod_{j=1}^{l(g)} u^{r_{i_{j}}}
$$

and $r_{i_{j}}$ is the number of times bond $i_{j}$ is traversed by $p_{1}, \ldots, p_{k}$, that is, If $p_{1}, \ldots, p_{k}$ traverse the $i$-th bond $m_{1}(i), \ldots, m_{k}(i)$ times, $m \geq 0$, respectively, then

$$
r_{i_{j}}=\sum_{a=1}^{k} m_{a}\left(i_{j}\right)
$$


Some but not all of the $m$ 's can be zero so that $r_{i j} \geq 1$ with at least one $r_{i j}>1$.

Let's consider the set of all terms with the same effective set of bonds $\{i\}$ traversed, hence, the terms associated to a given $g$. Within this set it's possible in general to find terms with the same powers $\{r\}$ and the same $\left|w_{g}\right|$ although having distinct associated paths and possibly with different effective sign.

Let's group together those terms which cover the same bonds of $g$ the same number of times. Denote by $\mathcal{W}_{g, N}(r)$ the set of terms $w_{g}$ with the same powers $\left\{r_{i_{j}}\right\}$ and such that $\sum_{j=1}^{l(g)} r_{i_{j}}=N$, for fixed $N$. The summation over all terms with repeated lines can now be expressed as

$$
\sum_{g \in \mathcal{G}} \sum_{\{N\}_{g}} \sum_{r(N)} \sum_{w_{g} \in \mathcal{W}_{g, N}(r)} w_{g}
$$

where $\sum_{g \in \mathcal{G}}$ means summation over all elements in $\mathcal{G}$; $\sum_{\{N\}_{g}}$ means summation over all positive integers $N$ compatible to the given graph $g$ and such that $N \geq l(g)+1$; $\sum_{r(N)}$ means summation over a set of positive integers $r_{1}, \ldots, r_{l}$ such that $r_{1}+\ldots+r_{l}=N$ and which are also compatible to $g$; and, finally, $\sum_{w_{g}}$ means summation over all terms $w_{g} \in \mathcal{W}_{g, N}(r)$.

Now the following remarks come to order. In the second summation, the case $N=l$ is excluded for it implies that $r_{i}=1$ and in this case there can be no repeated bonds. The case $N<l$ corresponds to another element $g^{\prime} \in \mathcal{G}$. The equality depends on the graph $g$. For instance, take the graph shown in Fig. 1b where $l+1=9$. No nonperiodic closed path with repeated bonds can have length $N=9$ because $l(g)=8$. The length $N$ can only be even and its minimum is $N=12$. Hence, for this particular graph $g$ the summation is over all even numbers greater or equal to 12 . In any case, the set $\{N\}_{g}$ has always infinite elements. Given $g$ and $N \in\{N\}_{g}$, not all partitions of $N$ are allowed in the third sum. For instance, given the graph in Fig. $1 \mathrm{~b}$ and $N=12$, the partition with $r_{i_{k}}=1, \forall k \neq 1$, and $r_{i}=5$ can not be associated to any allowed path. So, the set of integers $\{N\}$ and partitions of $N$ must be suitable to each $g$.

Given $g$, let's consider now the partial sums

$$
s_{n}=\sum_{\{N \mid N \leq n\}_{g}} \sum_{r(N)} \sum_{w_{g} \in \mathcal{W}_{g, N}(r)} w_{g}
$$

The goal is to show that in the limit $n \rightarrow \infty, s_{n}$ goes to zero. In ref. [7] its proved that $s_{n}=0$. The argument of the proof goes as follows.

Since the bonds of $g$ are covered the same number of times by all elements in the group, choose a bond of $g$, say $b$, which is traversed $\bar{r}>1$ times by all elements in the group $\mathcal{W}_{g, N}$. This choice has to be done for each partition $r(N)$. Denote by $P$ the set of paths associated to $w_{g}$. Then, $P=P^{\prime} \cup P^{\prime \prime}$ where $P^{\prime}$ is the set of those paths which traverse bond $b$ whereas $P^{\prime \prime}$ is the set of those paths which do not traverse $b$.
Given a path $p \in P^{\prime}$, let $p_{c}$ be the path segment obtained from $p$ upon removal of $b$. Given $P^{\prime}=\left\{p, p^{\prime} \ldots\right\}$ define

$$
P_{c}^{\prime}=\left\{p_{c}, p_{c}^{\prime}, \ldots\right\}
$$

This set has exactly $\bar{r}$ path segments. Collect under a same subgroup $S$ the elements $w_{g} \in \mathcal{W}_{g, N}$ having the property that line $b$ is covered exactly $\bar{r}$ times by all elements in $S$ and they all have the same subset $P_{c}^{\prime}$ with $\bar{r}$ path segments and the same subset $P^{\prime \prime}$. The set $\mathcal{W}_{g, N}$ is the union of such subsets, that is,

$$
\sum_{w_{g} \in \mathcal{W}_{g, N}(r)} w_{g}=\sum_{S \subset \mathcal{W}_{g, N}(r)} \sum_{w_{g} \in S} s(w)\left|w_{g}\right|
$$

where $s(w)$ is the sign of $w_{g}$ and $\left|w_{g}\right|=u^{N}$. Recall that $|u|<1$ so that $\left|w_{g}\right|<1$.

The elements inside any given $S$ cancel each other. Denote by $q$ and $e$ the elements of $S$ that are in $P^{\prime}$ and $P^{\prime \prime}$, respectively. Suppose that the segments $q_{1}, \ldots, q_{\bar{r}}$ are all distinct. (For the case with repeated segments, see [7]). The terms in $S$ are precisely those which can be obtained by joining the ends of the segments and this can be done in exactly $\bar{r}$ ! ways. This gives the possible terms $w_{g}$ in the subgroup. From the properties of the permutation group half of $N$ ! permutations are odd and half are even and so the signs of half of the terms are positive and half are negative, hence, a cancellation takes place.

Using (3.14), the partition function of the two dimensional Ising model can now be expressed as a product over paths as follows:

$$
Z_{N}(u)=2^{N^{2}}\left(1-u^{2}\right)^{-N(N-1)} \prod_{[p]}\left[1+W_{p}(u)\right]
$$

The next step consists in expressing the product over $[p]$ as an integral. This will be achieved in the next section.

\section{Paths amplitudes and Onsager's formula}

Consider all paths that start at a fixed site $P_{1}$ which we take as the origin with coordinates $(0,0)$ and end at the site $P_{n+1}$ with coordinates $(x, y)$ in $n$ steps. Starting at $(0,0)$ and whenever a site is reached there are four possible directions which a path can take (see Figure 2 and the Remark below). The path a) continues forward in the same direction of the previous step; b) it turns left $90^{\circ}$ relative to the previous step; c) it turns right $90^{\circ}$ relative to the previous step; d) it turns $180^{\circ}$. To each one of this possibilities it is assigned an "amplitude" which is given by: A) $u$ for the case a); B) $u \alpha$ for the case b); C) $u \bar{\alpha}$ for the case c) and D) 0 (zero) for the case d), where $u=\operatorname{tgh} K$ and $\alpha=e^{i \pi / 4}$ is the contribution to the sign of $p$ each time it turns left (counterclockwisely) relative to the previous step and $\bar{\alpha}$, when it turns right (clockwisely). See the Remark after Example 1, sec. 3.2 . 


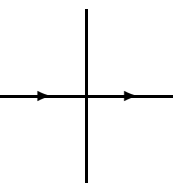

(a)

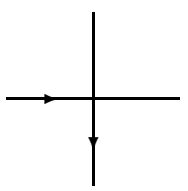

(c)

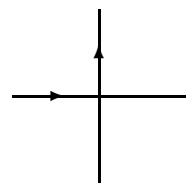

(b)

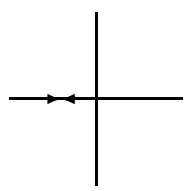

(d)
Figure 2: Directions which a path can take

at a valence 4 site.

Remark. The lattice being finite it has a border so that when a path strikes a site on the border it may have there only two or three possible directions to follow. In the spirit of refs. [7,9] we shall neglect the border and derive the relevant formulas as if there was no border at all with the justification that in the limit $N \rightarrow \infty$ which we shall take in the end of the calculation border effects dissapear. Of course, another approach would be to do everything on a toroidal lattice. In this case, however, relation (3.14) must be replaced by another more involved identity apropriate for the toroidal lattice ( given in refs. $[4,10]$ ). We shall restrict the presentation to the planar case only.

Call $U_{n}(x, y)$ the amplitude of arrival at $(x, y)$ moving upward in the $n$-th step, $D_{n}(x, y)$ the amplitude of arrival at $(x, y)$ moving downwards in the $n$-th step, $L_{n}(x, y)$ the amplitude of arrival at $(x, y)$ moving from the left in the $n$-th step, and $R_{n}(x, y)$ the amplitude of arrival at $(x, y)$ moving from right in the $n$-th step.

If the path arrives at $(x, y)$ moving upward in the n-th step then

$$
\begin{aligned}
& U_{n}(x, y)=u U_{n-1}(x, y-1)+0 D_{n-1}(x, y-1) \\
& +u \alpha L_{n-1}(x, y-1)+u \bar{\alpha} R_{n-1}(x, y-1)
\end{aligned}
$$

where $U_{n-1}, D_{n-1}, L_{n-1}$ and $R_{n-1}$ are the amplitudes associated to the four possibilities to reach site $(x, y-1)$. Relation (4.1) can be understood as follows. If $(x, y-1)$ is reached going up a bond in the $(n-1)$-th step, there the amplitude is $U_{n-1}(x, y-1)$ so in the $n$-th step as the path follows the same direction of the previous step, by the rules a) and $A$ ) above, a factor $u$ is multiplied to the amplitude $U_{n-1}(x, y-1)$. See Figure 3 .

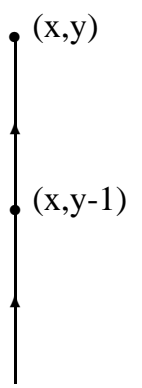

Figure 3: $p$ goes up to $(x, y-1)$ and $(x, y)$ in the $(n-1)$-th and $n$-th steps.

If the site $(x, y-1)$ is reached from the left in the $(n-1)$ th step (Figure 4), the path has to make a counterclockwise rotation to go to $(x, y)$ in the $n$-th step. By the rules $b)$ and $B)$ a factor $u \alpha$ should then be multiplied to the amplitude $L_{n-1}(x, y-1)$.

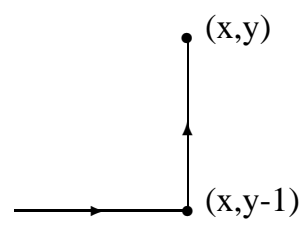

Figure 4. $p$ turns counterclockwise at $(x, y-1)$ to go to $(x, y)$ in the $\mathrm{n}$-th step.

The case that the path goes down to $(x, y-1)$ in the $(n-1)$ th step and goes up to $(x, y)$ in the $n$-th step corresponds to a $180^{\circ}$ rotation. By rules $d$ ) and $D$ ) the amplitude should be $0 . D_{n-1}(x, y-1)$. If the site $(x, y-1)$ is reached from the right the path has to make a clockwise rotation to go to $(x, y)$ (Figure 5). By the rules $c)$ and $C$ ) a factor $u \bar{\alpha}$ should then be multiplied to the amplitude $R_{n-1}(x, y-1)$.

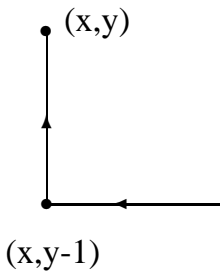

Figure 5. $p$ turns clockwise at $(x, y-1)$ to go to $(x, y)$ in the $\mathrm{n}$-th step.

Analogously, if a path arrives at $(x, y)$ in the $n$-th step going down the amplitude is given by the relation

$$
\begin{aligned}
& D_{n}(x, y)=0 U_{n-1}(x, y+1)+u D_{n-1}(x, y+1) \\
& +u \bar{\alpha} L_{n-1}(x, y+1)+u \alpha R_{n-1}(x, y+1)
\end{aligned}
$$


If it arrives at $(x, y)$ coming from the left then the amplitude is given by

$$
\begin{gathered}
L_{n}(x, y)=u \bar{\alpha} U_{n-1}(x-1, y)+u \alpha D_{n-1}(x-1, y) \\
+u L_{n-1}(x-1, y)+0 R_{n-1}(x-1, y)
\end{gathered}
$$

At last, if it arrives at $(x, y)$ coming from the right the amplitude is

$$
\begin{gathered}
R_{n}(x, y)=u \alpha U_{n-1}(x+1, y)+u \bar{\alpha} D_{n-1}(x+1, y) \\
+0 L_{n-1}(x+1, y)+u R_{n-1}(x+1, y)
\end{gathered}
$$

Of course to compute an amplitude using the above recursion relations it is needed the amplitude in the zero-th step. We shall follow the convention of reference [10], namely, that in the zero-th step a path arrives at the origin moving upward so that $U_{0}(x, y)=\delta_{x, 0} \delta_{y, 0}$ and $D_{0}=R_{0}=L_{0}=0$. The amplitude to arrive in zero steps is one if the path arrives going upward at the origin and zero for any other point or any other direction of arrival.

Example 2. See Figure 6. Let's compute the amplitude of arrival at site $(2,1)$ in 3 steps moving upward in the third step. Only one path is possible in this case. Using the recursion (4.1),

$$
\begin{gathered}
U_{3}(2,1)=u U_{2}(2,0)+0 D_{2}(2,0) \\
+u \alpha L_{2}(2,0)+u \bar{\alpha} R_{2}(2,0)
\end{gathered}
$$

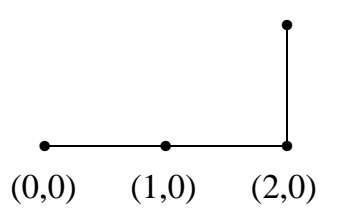

Figure 6: The path in Ex. 2.

In the second step, the path moves to site $(2,0)$ coming from the left so $U_{2}=D_{2}=R_{2}=0$ and $U_{3}(2,1)=u \alpha L_{2}(2,0)$. From (4.3),

$$
\begin{gathered}
L_{2}(2,0)=u \bar{\alpha} U_{1}(1,0) \\
+u \alpha D_{1}(1,0)+u L_{1}(1,0)+0 R_{1}(1,0)
\end{gathered}
$$

with $U_{1}=D_{1}=R_{1}=0$ so that $U_{3}(2,1)=u^{2} \alpha L_{1}(1,0)$ where

$$
\begin{gathered}
L_{1}(1,0)=u \bar{\alpha} U_{0}(0,0)+u \alpha D_{0}(0,0) \\
+u L_{0}(0,0)+0 R_{0}(0,0)=u \bar{\alpha}
\end{gathered}
$$

implying that $U_{3}(2,1)=u^{3}$.

Example 3. Let's now compute the amplitude of arrival at $(2,1)$ in 3 steps moving from the left in the third step. In this case, the possible paths are shown in Figure 7a) and 7b).

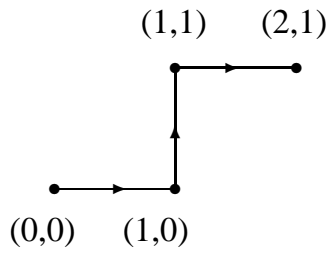

(a)
$(0,1) \quad(1,1) \quad(2,1)$

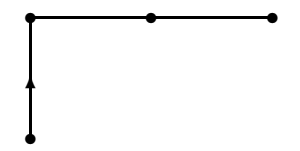

$(0,0)$

(b)
Figure 7: The paths in Ex. 3.

Using relation (4.3), the amplitude is

$$
\begin{gathered}
L_{3}(2,1)=u \bar{\alpha} U_{2}(1,1) \\
+u \alpha D_{2}(1,1)+u L_{2}(1,1)+0 R_{2}(1,1)
\end{gathered}
$$

Using (4.1),

$$
\begin{gathered}
U_{2}(1,1)=u U_{1}(1,0)+0 D_{1}(1,0) \\
+u \alpha L_{1}(1,0)+u \bar{\alpha} R_{1}(1,0)
\end{gathered}
$$

Since $U_{1}=D_{1}=R_{1}=0$, one finds that $U_{2}(1,1)=$ $u \alpha L_{1}(1,0)=u \alpha u \bar{\alpha}=u^{2}$. Using (4.3), with $D_{1}=L_{1}=$ $R_{1}=0$,

$$
\begin{gathered}
L_{2}(1,1)=u \bar{\alpha} U_{1}(0,1)+u \alpha D_{1}(0,1) \\
+u L_{1}(0,1)+0 R_{1}(0,1)=u \bar{\alpha} u
\end{gathered}
$$

Therefore, $L_{3}(2,1)=2 u^{3} \bar{\alpha}$.

Definition 4.1. The partial amplitude of a path $p$ of length $n$ is given by

$$
\bar{W}_{p}(u)=\prod \alpha \prod \bar{\alpha} u^{n}
$$

Definition 4.2 . The amplitude $\sum_{p} \bar{W}_{p\left(n, P_{1}\right)}(u)$ of arrival at $P_{n+1}(x, y)$ from any direction in $n$ steps is given by

$$
U_{n}(x, y)+D_{n}(x, y)+L_{n}(x, y)+R_{n}(x, y)
$$

Example 4. The partial amplitudes for the paths in Figure 6, 7a) and 7b) are $u^{3}, \bar{\alpha} u^{3}$ and $\bar{\alpha} u^{3}$, respectively. The amplitude of arrival at $(2,1)$ from any direction in 3 steps is, then, $u^{3}+2 \bar{\alpha} u^{3}$.

Definition 4.3. Fix $n$ and call $C_{n}(x, y)$ the set of all paths of length $n$ starting at $(0,0)$ and arriving at $(x, y)$. Given $p \in C_{n}$ and $F_{n} \in \mathcal{B}(x, y)$ where

$$
\mathcal{B}(x, y)=\left\{U_{n}(x, y), D_{n}(x, y), L_{n}(x, y), R_{n}(x, y)\right\}
$$

Define the extension of $F_{n}(x, y)$, denoted by the same symbol, so as to include sites $(x, y)$ which can be reached only by a number $m>n$ of steps but in this case set $F_{n}(x, y)=0$.

Lemma. The transform of $F_{n}$, the function $\bar{F}_{n}(\epsilon, \eta), 0 \leq$ $\epsilon \leq 2 \pi$ and $0 \leq \eta \leq 2 \pi$, given by

$$
\bar{F}_{n}(\varepsilon, \eta)=\sum_{x=-\infty}^{\infty} \sum_{y=-\infty}^{\infty} F_{n}(x, y) e^{-i \varepsilon x} e^{-i \eta y}
$$


is well defined and

$$
F_{n}(x, y)=\int_{0}^{2 \pi} \int_{0}^{2 \pi} e^{i \varepsilon x} e^{i \eta y} \bar{F}_{n}(\varepsilon, \eta) \frac{d \varepsilon d \eta}{(2 \pi)^{2}}
$$

Proof: $F_{n}(x, y)=0$ for $|x|>n$ or/and $|y|>n$. Then, for fixed $n$ the sums in (4.14) have only a finite number of terms.

Using (4.14), the transform of $U_{n}(x, y)$ is:

$$
\bar{U}_{n}(\varepsilon, \eta)=\sum_{x=-\infty}^{\infty} \sum_{y=-\infty}^{\infty} U_{n}(x, y) e^{-i \varepsilon x} e^{-i \eta y}
$$

Upon substitution of (4.1), and making the change $\bar{y}=y-1$ it follows that

$$
\begin{gathered}
\bar{U}_{n}(\varepsilon, \eta)=u e^{-i \eta} \bar{U}_{n-1}(\varepsilon, \eta) \\
+0 \bar{D}_{n-1}(\varepsilon, \eta)+u \alpha e^{-i \eta} \bar{L}_{n-1}(\varepsilon, \eta)+u \bar{\alpha} e^{-i \eta} \bar{R}_{n-1}(\varepsilon, \eta)
\end{gathered}
$$

Similarly, we obtain $\bar{D}_{n}(\varepsilon, \eta), \bar{L}_{n}(\varepsilon, \eta)$ and $\bar{R}_{n}(\varepsilon, \eta)$ :

$$
\begin{aligned}
& \bar{D}_{n}(\varepsilon, \eta)=0 \bar{U}_{n-1}(\varepsilon, \eta)+u e^{i \eta} \bar{D}_{n-1}(\varepsilon, \eta) \\
& +u \bar{\alpha} e^{i \eta} \bar{L}_{n-1}(\varepsilon, \eta)+u \alpha e^{i \eta} \bar{R}_{n-1}(\varepsilon, \eta)
\end{aligned}
$$

$$
\begin{gathered}
\bar{L}_{n}(\varepsilon, \eta)=u \bar{\alpha} e^{-i \varepsilon} \bar{U}_{n-1}(\varepsilon, \eta)+u \alpha e^{-i \varepsilon} \bar{D}_{n-1}(\varepsilon, \eta) \\
+u e^{-i \varepsilon} \bar{L}_{n-1}(\varepsilon, \eta)+0 \bar{R}_{n-1}(\varepsilon, \eta) \\
\bar{R}_{n}(\varepsilon, \eta)=u \alpha e^{i \varepsilon} \bar{U}_{n-1}(\varepsilon, \eta)+u \bar{\alpha} e^{i \varepsilon} \bar{D}_{n-1}(\varepsilon, \eta) \\
+0 \bar{L}_{n-1}(\varepsilon, \eta)+u e^{i \varepsilon} \bar{R}_{n-1}(\varepsilon, \eta)
\end{gathered}
$$

Call $\psi_{n}(\epsilon, \eta)$ the matrix

$$
\psi_{n}=\left(\begin{array}{llll}
\bar{U}_{n} & \bar{D}_{n} & \bar{L}_{n} & \bar{R}_{n}
\end{array}\right)
$$

Then, from (4.17-20) we obtain that

$$
\psi_{n}(\varepsilon, \eta)=\psi_{n-1}(\varepsilon, \eta) u M
$$

where

$$
M=\left(\begin{array}{cccc}
v & 0 & \overline{\alpha h} & \alpha h \\
0 & \bar{v} & \alpha \bar{h} & \bar{\alpha} h \\
\alpha v & \overline{\alpha v} & \bar{h} & 0 \\
\bar{\alpha} v & \alpha \bar{v} & 0 & h
\end{array}\right)
$$

with $v=e^{-i \eta}, \bar{h}=e^{-i \varepsilon}, h=e^{i \varepsilon}, \bar{v}=e^{i \eta}$ and $\alpha=e^{i \frac{\pi}{4}}$.

Call $1,2,3$ and 4 the directions shown in the Figure 8 below:

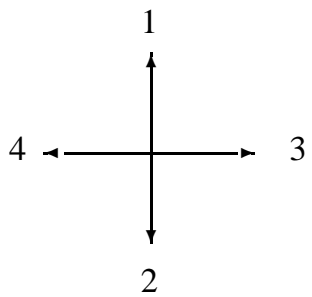

Figura 8. Directions associated to $M_{i j}$.
Notice that the subindices $i, j$ of $M_{i j}$ are in one-to-one with the directions. Indeed, $u M_{1 j}$ corresponds to the amplitude of arrival at $(x, y)$ (in $(\epsilon, \eta)$ space) coming up in the $(n-1)$ th step, $\forall j$, but going up if $j=1$, down if $j=2$, coming from the left if $j=3$ and coming from the right if $j=4$ in the $n$-th step. Therefore, $u M_{1 j}$ is the amplitude of arrival at $(x, y)$ (in $(\epsilon, \eta)$ space ) following directions 1 and $j$ in the $(n-1)$-th and $n$-th steps, respectively. More generally, $u M_{i j}$ is the amplitude of arrival at $(x, y)$ following directions $i$ and $j$ in the $(n-1)$-th and $n$-th steps, respectively. From now on only closed paths starting at $(0,0)$ and arriving at $(0,0)$ in $n$ steps will be considered. From (4.22) it follows that

$$
\begin{gathered}
\psi_{n}(\varepsilon, \eta)=\psi_{n-1}(\varepsilon, \eta)(u M) \\
=\psi_{n-2}(\varepsilon, \eta)(u M)^{2}=\cdots=\psi_{0}(u M)^{n}
\end{gathered}
$$

Denote by $\psi_{0, i}, 1 \leq i \leq 4$, the line matrix with the only element distinct from zero and equal to 1 in the $i$-th column. Let $\psi_{0} \equiv \psi_{0, i}$ according to whether the path arrives at the origin moving up $(i=1)$, down $(i=2)$, from the left $(i=3)$ or from the right $(i=4)$, respectively. Then

$$
\bar{F}_{n}(\varepsilon, \eta)=\psi_{0, i}(u M)^{n} \psi_{0, i}^{T}
$$

where $i=1,2,3,4$ if $F=U, D, L, R$, respectively, and $\Psi^{T}$ is the transpose of $\Psi$ and

$$
\sum_{F_{n} \in B_{n}} \bar{F}_{n}(\epsilon, \eta)=\sum_{k=1}^{4} \psi_{0, k}(\varepsilon, \eta)(u M)^{n} \psi_{0, k}^{T}(\varepsilon, \eta)
$$

Given a $4 \times 4$ matrix $A, \psi_{0, i} A$ is the line matrix formed by the elements in the $i$-th line of $A$, that is,

$$
\psi_{0, i} A=\left(\begin{array}{cccc}
A_{i, 1} & A_{i, 2} & A_{i, 3} & A_{i, 4}
\end{array}\right)
$$

so $\psi_{0, i} A \psi_{0, i}^{T}=A_{i i}$. Therefore, the sum over $i$ equals the trace of $A$. Thus,

$$
\sum_{k=1}^{4} \psi_{0, k}(u M)^{n} \psi_{0, k}^{T}=\operatorname{Tr}(u M)^{n}
$$

The total partial amplitude of arrival at $(0,0)$ of closed paths moving in any direction in $n$ steps given by (4.12) can be expressed compactly as

$$
\sum_{F_{n} \in B_{n}} F_{n}(0,0)
$$

From (4.15), (4.26) and (4.28), it follows that

$$
\sum_{F_{n} \in B_{n}} F_{n}(0,0)=\int_{0}^{2 \pi} \int_{0}^{2 \pi} \operatorname{Tr}(u M)^{n} \frac{d \varepsilon d \eta}{(2 \pi)^{2}}
$$

To better understand relation (4.30), consider the matrix $u^{n} M^{n}$, for some $n$. An element $\left(u^{n} M^{n}\right)_{i_{1} i_{n+1}}$ of this matrix is given as 


$$
\left(u^{n} M^{n}\right)_{i_{1} i_{n+1}}=\sum_{i_{2}, \ldots, i_{n}=1}^{4} u M_{i_{1} i_{2}} u M_{i_{2} i_{3}} \ldots u M_{i_{n-1} i_{n}} u M_{i_{n} i_{n+1}} .
$$

Recall that $u M_{i, j}$ is the partial amplitude of a path arriving at a site coming from direction $i$ and going to the next site in one step following direction $j$. Thus, each term in the r.h.s. of (4.31) is the amplitude of a path of length $n$ starting at $P_{1}$ coming from direction $i_{1}$, going to $P_{2}$ following direction $i_{2}$, etc, and arriving at site $P_{n+1}$ following direction $i_{n+1}$ after $n$ steps. The element $\left(u^{n} M^{n}\right)_{i_{1} i_{n+1}}$ gives the total partial amplitude of arrival at $P_{n+1}$ in $n$ steps in $(\epsilon, \eta)$ space.

The terms in $\left(u^{n} M^{n}\right)_{i_{1} i_{n+1}}$ describe open as well as closed paths. Let's see some examples.

Example 5. Take $n=5, i_{1}=2$ and $i_{6}=1$. The term $M_{23} M_{31} M_{11} M_{14} M_{41}$ describes a path beginning at $P_{1}$ where it arrived coming from direction $i_{1}=2$, going to $P_{2}, P_{3}, P_{4}, P_{5}$ and to $P_{6}$ following directions $i_{2}=3$, $i_{3}=1, i_{4}=1, i_{5}=4$ and $i_{6}=1$, respectively. See Figure 9a).

Example 6. Take $n=6, i_{1}=i_{6}=2$ and the term $M_{23} M_{31} M_{11} M_{14} M_{42} M_{22}$ of $\left(M^{6}\right)_{22}$. This term describes the closed path in Fig. 9b. The elements of $M^{n}$ outside the diagonal have associated to them only open paths. This is implied by the simple fact that these elements have $i_{1} \neq i_{n+1}$. Closed paths are to be found only in the diagonal elements since there $i_{1}=i_{n+1}$. However, open paths can also be associated to some terms in the diagonal elements. Let's see some examples.

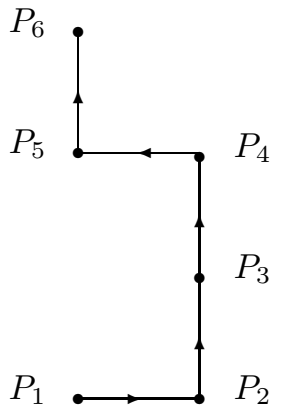

(a)

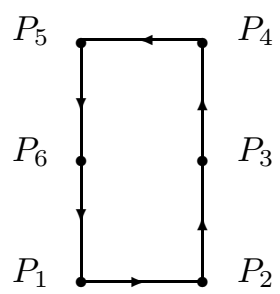

(b)
Figure 9: Paths in (a) Ex. 5 and in (b) Ex. 6.

Example 7. Take $n=2, i_{1}=i_{3}=1$ and the element $\left(M^{2}\right)_{11}=M_{11} M_{11}+M_{12} M_{21}+M_{13} M_{31}+M_{14} M_{41}$ with

$$
u^{2}\left(M^{2}\right)_{11}=u^{2} v^{2}+0+u^{2}(\bar{\alpha} \bar{h})(\alpha v)+u^{2}(\alpha h)(\bar{\alpha} v)
$$

To each one of the terms of $\left(M^{2}\right)_{11}$ correspond the paths (a), (b), (c) and (d), respectively, shown in Figure 10.

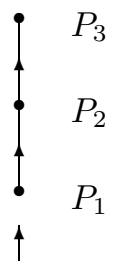

(a)

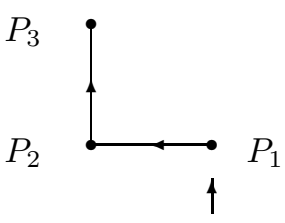

(c)

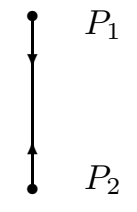

(b)

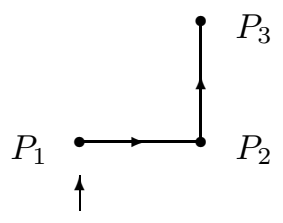

(d)
Figure 10: Paths in $\left(M^{2}\right)_{11}$

Example 8. Take $n=4$ and consider the following terms in $\left(M^{4}\right)_{11}$ :

a)The term $u^{4} M_{11} M_{11} M_{11} M_{11}=u^{4} v^{4}$ is the amplitude of the open path shown in fig. 11 below.

$$
i
$$

Figure 11: Path $\left(M_{11}\right)^{4}$.

b) The term $M_{11} M_{11} M_{13} M_{31}=v v(\bar{\alpha} \bar{h})(\alpha v)=v^{3} \bar{h} v$ whose associated open path is shown in Figure 12. 


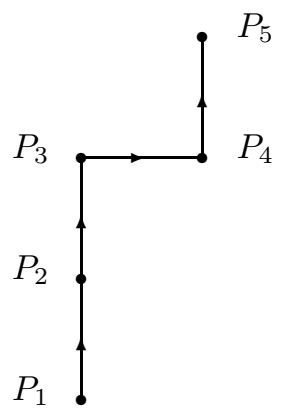

Figure 12: Path $M_{11} M_{11} M_{13} M_{31}$.

c) The term $u^{4} M_{13} M_{32} M_{24} M_{41}=u^{4}(\bar{\alpha} \bar{h})(\overline{\alpha v})(\bar{\alpha} h)(\bar{\alpha} v)=$ $u^{4} \bar{\alpha}^{4}(\bar{h} h)(\bar{v} v)=u^{4} \bar{\alpha}^{4}$ is the amplitude of the closed path shown in Fig. 13:

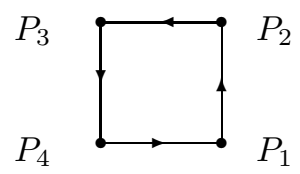

Figure 13: Path $M_{13} M_{32} M_{24} M_{41}$.

In order to restrict to the elements of $M^{n}$ having closed paths we must take the trace of $M^{n}$. A closed path begins at and return to $P_{1}$ after $n$ steps. Since it is closed it has to cover $n / 2$ horizontal bonds in one direction and $n / 2$ horizontal bonds following the opposite direction. The same is true for the vertical bonds traversed by $p$. So, if the term $M_{i_{1} i_{2}} M_{i_{2} i_{3}} \ldots M_{i_{n-1} i_{n}} M_{i_{n} i_{n+1}}, i_{n+1}=i_{n}$, describes a closed path, then the number of $h$ 's ( $v$ 's) equals the number of $\bar{h}$ 's ( $\bar{v}$ 's) appearing in it. In this case, it's possible to organize the term into a product of pairs $h \bar{h}=1$ and $v \bar{v}=1$ and the double integral in $\epsilon$ and $\eta$ will give $(2 \pi)^{2}$ times a product of $\alpha$ 's and $\bar{\alpha}$ 's. More precisely, the double integral over a closed path in $\operatorname{Tr} M^{n}$ equals

$$
(2 \pi)^{2} \prod \alpha \prod \bar{\alpha}
$$

where the first product is over all counterclockwise rotations and the second is over all clockwise rotations, so

$$
\prod \alpha \prod \bar{\alpha}=(-1)^{t(p)}
$$

where $t(p)$ is the number of complete $2 \pi$ revolutions performed by a tangent vector traversing the closed path $p$. Remind that one has yet to multiply $(4.34)$ by $(-1)$ in order to get the complete sign $s(p)$ of $p$.

If a path is open the $h$ 's, $\bar{h}$ 's ( $v$ 's, $\bar{v}$ 's) don't match up into pairs. There will be left integrals of the form

$$
\int_{0}^{2 \pi} e^{i k \theta} d \theta=0
$$

where $\theta$ stands for $\eta$ or $\epsilon$ and $k \geq 1$, hence, the integrals in $\eta$ and $\varepsilon$ remove completely terms describing open paths. Let's see examples.

Example 9. Take $n=1$. In this case there are only open paths and

$$
\int_{0}^{2 \pi} \int_{0}^{2 \pi} d \eta d \varepsilon M_{i j}=0, \quad \forall i, j
$$

Example 10. Using (4.35), in ex. 7,

$$
\int_{0}^{2 \pi} \int_{0}^{2 \pi} d \eta d \varepsilon\left(M^{2}\right)_{11}=0
$$

Example 11. It is clear that

$$
\int d \varepsilon d \eta\left(M^{n}\right)_{i j}=0 \quad \forall i, j
$$

if $n=1,2,3$, which is guaranteed by the fact that in a square lattice closed paths are possible only if $n \geq 4$. In the case $n=2$ the path in Figure 10.b) is closed but it traverses the same edge back and its amplitude is thus zero.

Example 12. Using (4.35) in ex.8, for the term $M_{11} M_{11} M_{11} M_{11}=v^{4}$

$$
\int_{0}^{2 \pi} \int_{0}^{2 \pi} v^{4} d \eta d \varepsilon=2 \pi \int_{0}^{2 \pi} e^{-4 i \eta} d \eta=0 .
$$

For the term $M_{11} M_{11} M_{13} M_{31}=v^{3} \bar{h}$,

$$
\int_{0}^{2 \pi} d \eta d \varepsilon v^{3} \bar{h}=0
$$

For the term $M_{13} M_{32} M_{24} M_{41}=\bar{\alpha}^{4}$ which describes a closed path the result

$$
\frac{1}{(2 \pi)^{2}} \int_{0}^{2 \pi} d \eta d \varepsilon \bar{\alpha}^{4}=\bar{\alpha}^{4} .
$$

follows which has the form (4.33-34) with $\bar{\alpha}^{4} \equiv 1$.

Given a closed path in $\left(M^{n}\right)_{i i}$, the inverse path is present in some $\left(M^{n}\right)_{j j}, j \neq i$. For instance, in $\left(M^{4}\right)_{11}$ there are the closed paths shown in Fig. 14 given by the terms $M_{14} M_{42} M_{23} M_{31}$ and $M_{13} M_{32} M_{24} M_{41}$.

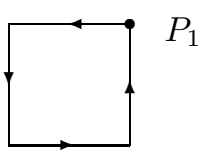

(a)

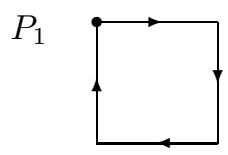

(b)
Figure 14: Paths (a) $M_{14} M_{42} M_{23} M_{31}$ and (b) $M_{13} M_{32} M_{24} M_{41}$. 
In $\left(M^{4}\right)_{22}$ there are the terms $M_{24} M_{41} M_{13} M_{32}$ and $M_{23} M_{31} M_{14} M_{42}$, with associated closed paths shown in Fig. $15 \mathrm{c}$ and $15 \mathrm{~d}$, respectively:

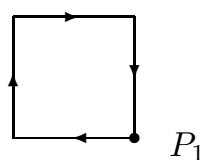

(c)

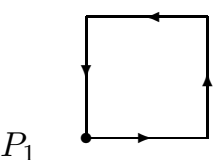

(d)
Figura 15: Paths (c) $M_{24} M_{41} M_{13} M_{32}$ and (d) $M_{23} M_{31} M_{14} M_{42}$

In $\left(M^{4}\right)_{33}$, there are the terms $M_{32} M_{24} M_{41} M_{13}$ and $M_{31} M_{14} M_{42} M_{23}$ with associated closed paths shown in Figure 16e) and 16f), respectively.

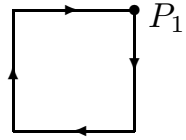

(e)

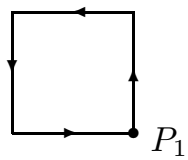

(f)
Figura 16. Paths (e) $M_{32} M_{24} M_{41} M_{13}$ and (f) $M_{31} M_{14} M_{42} M_{23}$

In $\left(M^{4}\right)_{44}$, there are the terms $M_{42} M_{23} M_{31} M_{14}$ and $M_{41} M_{13} M_{32} M_{24}$ with the associated closed paths shown in Figure $17 \mathrm{~g}$ ) and $17 \mathrm{~h}$ ), respectively.

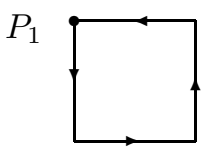

$(\mathrm{g})$

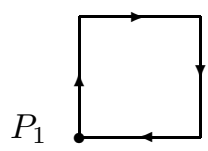

(h)
Figura 17. Paths (g) $M_{42} M_{23} M_{31} M_{14}$ and (h) $M_{41} M_{13} M_{32} M_{24}$

Note that (e) is the inversion of (a), (f) is the inversion of (c), (g) of (b), and (h) of (d).

So restricting to the diagonal terms of $M^{n}$ which amounts to take the trace of this matrix and then performing a double integration on the angles to eliminate open paths, dividing the result by 2 to eliminate inversions, and multiplying the result by $-u^{n}$ gives the total complete amplitude
( with the right signs ) to arrive back at $P_{1}$ in $n$ steps moving in any direction. We have thus achieved the following relation:

$$
\sum_{p\left(n, P_{1}\right)} W_{p}(u)=-\frac{1}{2} \frac{1}{(2 \pi)^{2}} \int_{0}^{2 \pi} \int_{0}^{2 \pi} d \varepsilon d \eta T r(u M)^{n}
$$

The above result is restricted to a fixed site $P_{1}$. For the finite $N \times N$ lattice with $N^{2}$ sites and disregarding boundary effects, the total (independent of site) amplitude of closed paths of length $n$ is:

$$
\begin{gathered}
N^{2} \sum_{p\left(n, P_{1}\right)} W_{p}(u) \equiv \sum_{p(n)} W_{p}(u) \\
=-\frac{N^{2}}{2} \frac{1}{(2 \pi)^{2}} \int_{0}^{2 \pi} \int_{0}^{2 \pi} d \varepsilon d \eta \operatorname{Tr}(u M)^{n}
\end{gathered}
$$

Taking all $N^{2}$ sites into account imply that given a closed path $p(n)$, the summation $\sum_{p(n)} W(p)$ includes all circular permutations of $p$. To eliminate these the previous relation has to be divided by $n$. Then, the amplitude is given by

$$
\frac{1}{n} \sum_{p(n)} W_{p}(u)=-\frac{N^{2}}{2} \frac{1}{(2 \pi)^{2}} \int_{0}^{2 \pi} \int_{0}^{2 \pi} d \varepsilon d \eta \frac{\operatorname{Tr}(u M)^{n}}{n}
$$

We notice that a nonperiodic path appears $n$ times in the sum but a periodic path of length $n$ and period $w$ has $n / w$ distinct starting points only and for this reason it appears $n / w$ times in the sum over paths. For instance, the periodic path $\left(D_{j_{1}} D_{j_{2}}\right)\left(D_{j_{1}} D_{j_{2}}\right)\left(D_{j_{1}} D_{j_{2}}\right)$ of length $n=6$ and period $w=3$ has only two distinct starting points. The other equivalent periodic path is $D_{j_{2}}\left(D_{j_{1}} D_{j_{2}}\right)\left(D_{j_{1}} D_{j_{2}}\right) D_{j_{1}}$. After division by $n$, periodic paths with period $w$ will show up in the sum with a weight $1 / w$. Thus, the above relation includes all closed paths of length $n$ over the $N \times N$ lattice, periodic and nonperiodic, and excludes inversions and circular permutations. The total amplitude of closed paths of any length is then given by the series

$$
\sum_{n} \frac{1}{n} \sum_{p(n)} W_{p}(u)=
$$

$$
\sum_{n=1}^{\infty}-\frac{N^{2}}{2} \frac{1}{(2 \pi)^{2}} \int_{0}^{2 \pi} \int_{0}^{2 \pi} d \varepsilon d \eta \frac{\operatorname{Tr}(u M)^{n}}{n}
$$

whose convergence will be investigated below. We note that since the lattice is square, closed paths with nonzero amplitude are possible only for $n>3$ but in view of relation (4.38) in Ex. 11 we can write the series in (4.45) starting from $n=1$.

With the above remarks,

$$
\sum_{n=1}^{\infty} \frac{1}{n} \sum_{p(n)} W_{p}(u) \equiv
$$




$$
\sum_{[p]}\left[W_{p}(u)-\frac{1}{2}\left(W_{p}(u)\right)^{2}+\frac{1}{3}\left(W_{p}(u)\right)^{3}-\ldots\right]
$$

In $\sum_{[p]}$ the first term is the sum of $W_{p}(u)$ over all nonperiodic paths. The other terms give the sum over all periodic paths since any periodic path is the repetition of some nonperiodic path $p$ with period given by $w=2,3, \ldots$ In section 3.2 the sign of a periodic path was proved to be -1 if $w$ is even and equal to the sign of its nonperiodic subpath if $w$ is odd. This explains the signs in the r.h.s of (4.46).

Since $|u|<1$ then $|W|<1$ and the series between brackets converges to $\ln (1+W)$, and the r.h.s. of (4.46) equals to

$$
\sum_{p} \ln \left(1+W_{p}(u)\right)=\ln \prod_{p}\left(1+W_{p}(u)\right) .
$$

a result to be used below.

Theorem 4.1. Take $|u| \leq r<\frac{1}{4}$. Then, the series

$$
\sum_{n=1}^{\infty} \frac{u^{n}\left(M^{n}\right)_{i j}}{n}
$$

converges uniformily.

Proof: We have that $\left|M_{i j}\right| \leq 1, \forall i, j=1,2,3,4$, so from (4.31) we get

$$
\left|\left(M^{n}\right)_{i j}\right| \leq 4^{n-1}
$$

and

$$
\left|\frac{u^{n}\left(M^{n}\right)_{i j}}{n}\right| \leq \frac{4^{n-1}|u|^{n}}{n}
$$

The series

$$
\sum_{n=1}^{\infty} \frac{(4|u|)^{n}}{n}
$$

converges for $|u| \leq r<\frac{1}{4}$, hence, by Weierstrass $M$-test the series (4.48) converges uniformly for $|u| \leq r<1 / 4$.

We may conclude that the series $\sum_{n=1}^{\infty} \frac{-(u M)^{n}}{n}$ converges uniformly to the matrix $\ln (1-u M)$ in the same interval.

We may now integrate the series term by term to get the series (4.45) which likewise converges uniformly in the same interval. Interchanging integration and summation in (4.45) yields

$$
\begin{aligned}
& \frac{N^{2}}{2} \frac{1}{(2 \pi)^{2}} \int_{0}^{2 \pi} \int_{0}^{2 \pi} d \varepsilon d \eta \operatorname{Tr} \sum_{n=1}^{\infty}-\frac{(u M)^{n}}{n} \\
= & \frac{N^{2}}{2} \frac{1}{(2 \pi)^{2}} \int_{0}^{2 \pi} \int_{0}^{2 \pi} d \varepsilon d \eta \operatorname{Tr} \ln (I-u M)
\end{aligned}
$$

From the previous analysis, $|u| \leq r<1 / 4$. However, the r.h.s. of (4.52) is well defined in a bigger domain. Using the relation

$$
\operatorname{Tr} \ln (I-u M)=\ln \operatorname{det}(I-u M)
$$

which is valid for $\operatorname{det}(1-u M) \neq 0$ [18], we get

$$
\frac{N^{2}}{2} \frac{1}{(2 \pi)^{2}} \int_{0}^{2 \pi} \int_{0}^{2 \pi} d \varepsilon d \eta \ln \operatorname{det}(I-u M)
$$

The determinant can be easily computed and one finds that

$$
\operatorname{det}(1-u M)=\left(u^{2}+1\right)^{2}-2 u\left(1-u^{2}\right)(\cos \varepsilon+\cos \eta)
$$

Taking the logarithm on both sides of relation (3.24) gives

$\frac{\ln Z(u)}{N^{2}}=\ln 2+2\left(1-\frac{1}{N}\right) \ln (\cosh K)+\ln \prod_{p}\left[1+W_{p}(u)\right]$

or, using (4.45-47), (4.52,4.54),

$$
\begin{gathered}
\frac{\ln Z(u)}{N^{2}}=\ln 2+2\left(1-\frac{1}{N}\right) \ln (\cosh K) \\
+\frac{1}{8 \pi^{2}} \int_{0}^{2 \pi} \int_{0}^{2 \pi} d \varepsilon d \eta \ln \operatorname{det}(1-u M)
\end{gathered}
$$

Using (4.55) with $u=\tanh K$ and the relations

$$
\begin{gathered}
u=\frac{1}{2} \sinh (2 K)\left(1-u^{2}\right) \\
\left(1+u^{2}\right)^{2}=\cosh ^{2}(2 K)\left(1-u^{2}\right)^{2} \\
\left(1-u^{2}\right)=\frac{1}{\cosh ^{2} 2 K}
\end{gathered}
$$

gives

$$
\begin{gathered}
\operatorname{det}(1-u M)=\cosh ^{-4} 2 K\left[(\cosh 2 K)^{2}\right. \\
-(\sinh 2 K)(\cos \eta+\cos \varepsilon)]
\end{gathered}
$$

Onsager's formula follows from (4.57) after taking the limit $N \rightarrow \infty$ :

$$
\begin{gathered}
-\frac{f}{k_{B} T}=\ln 2+\frac{1}{2 \pi^{2}} \int_{0}^{\pi} \int_{0}^{\pi} d \varepsilon d \eta \ln \left[(\cosh 2 K)^{2}\right. \\
-\sinh 2 K(\cos \eta+\cos \varepsilon)]
\end{gathered}
$$

where $f$ is the free energy per site in the thermodynamical limit ( see (2.5)).

The integral in (4.62) can not be evaluated in terms of simple functions. The derivatives of the integral however can be expressed in terms of elliptic functions [2,15,19].

Set $2 k=\tanh 2 K / \cosh 2 K$. Then,

$$
\begin{gathered}
-\frac{f}{k_{B} T}=\ln 2+\ln (\cosh 2 K) \\
+\frac{1}{2 \pi^{2}} \int_{0}^{\pi} \int_{0}^{\pi} d \varepsilon d \eta \ln [1-2 k(\cos \eta+\cos \varepsilon)]
\end{gathered}
$$

Expanding the logarithm in powers of $k$ it follows that

$$
-\frac{f}{k_{B} T}=\ln 2+\ln (\cosh 2 K)-\sum_{n=1}^{\infty}\left(\frac{(2 n) !}{(n !)^{2}}\right)^{2} k^{2 n}
$$

The series converges for $|2 k(\cos \epsilon+\cos \eta)| \leq 4|k|<1$. For $k>0(J>0)$ and at $k=1 / 4$, that is, at the critical value 
$K=K_{c}\left(\right.$ or temperature $\left.T_{c}=2 J k_{B}^{-1} l^{-1}(\sqrt{2}+1)\right)$ given by

$$
2 \operatorname{senh} 2 K_{c}=\cosh ^{2} 2 K_{c}
$$

it diverges. ( Similarly, for $J<0$ which implies $k<0$ and divergence at $\left.T_{c}=2 J k_{B}^{-1} l n^{-1}(\sqrt{2}-1)\right)$.

The internal energy $U$ is given by

$$
U=-k T^{2} \frac{\partial}{\partial T} \frac{f}{k T}
$$

From (4.63),

$$
U=-J \operatorname{coth}(2 K)\left[1+\left(\sinh ^{2} 2 K-1\right) I(2 K)\right]
$$

where

$$
I(2 K)=\frac{1}{\pi^{2}} \int_{0}^{\pi} \frac{d \epsilon d \eta}{\cosh ^{2}(2 K)-\sinh (2 K)(\cos \epsilon+\cos \eta)}
$$

By performing one of the integrals its found that

$$
U=-J \operatorname{coth}(2 K)\left[1+\left(2 \tanh ^{2} 2 K-1\right) \frac{2}{\pi} F\left(k_{1}\right)\right]
$$

where $k_{1}=4 k$ and $F\left(k_{1}\right)$ is the complete elliptic integral of the first kind defined by

$$
F\left(k_{1}\right)=\int_{0}^{\pi / 2}\left(1-k_{1}^{2} \operatorname{sen}^{2} \theta\right)^{-\frac{1}{2}} d \theta
$$

The elliptic function $F$ ( see Ref. [19] ) has the property that

$$
F \rightarrow \ln \left[4\left(1-k_{1}^{2}\right)\right]^{-1 / 2}
$$

as $k_{1} \rightarrow 1^{-}$. So it diverges logarithmically at $k_{1}=1$, or at the value $K_{c}$ given by (4.65).

In relation (4.69) for $U$ the function $F$ is multiplied by $\left(2 \tanh ^{2} 2 K-1\right)$ which is zero at the critical point $K_{c}$. Indeed, from the identity $\cosh ^{2} x=1+\sinh ^{2} x$ relation (4.65) implies that $\sinh \left(2 K_{c}\right)=1$. Using this and (4.65), $\tanh ^{2} 2 K_{c}=1 / 2$ follows. So the function $U$ is continuous at $K_{c}$.

The specific heat can be computed from the definition

$$
C=\frac{\partial U}{\partial T}
$$

It is given by

$$
\begin{gathered}
C=\frac{2 k_{1}}{\pi}(K \operatorname{coth} 2 K)^{2}\left\{2 F\left(k_{1}\right)-2 E\left(k_{1}\right)\right. \\
\left.+2\left(\tanh ^{2} 2 K-1\right) G\left(k_{1}\right)\right\}
\end{gathered}
$$

where

$$
G\left(k_{1}\right)=\frac{\pi}{2}+\left(2 \tanh ^{2} 2 K-1\right) F\left(k_{1}\right)
$$

and $E\left(k_{1}\right)$ is the complete elliptic integral of the second kind, defined by

$$
E\left(k_{1}\right)=\int_{0}^{\pi / 2}\left(1-k_{1}^{2} \operatorname{sen}^{2} \theta\right)^{\frac{1}{2}} d \theta
$$

which is well defined at $k_{1}=1$. From the exact result (4.73) it follows that the specific heat is logarithmically divergent at the critical point.

\section{References}

[1] E. Ising, Zeitschrift f. Physik 31, 253 (1925).

[2] L. Onsager, Phys. Rev. 65, 117 (1944).

[3] M. Kac and J. C. Ward, Phys. Rev. 88, 1332 (1952).

[4] S. Sherman, J. Math. Phys. 1, 202 (1960).

[5] S. Sherman, Bull. Am. Math. Soc. 68, 225 (1962).

[6] S. Sherman, J. Math. Phys. 4, 1213 (1963).

[7] P. N. Burgoyne, J. Math. Phys. 4, 1320 (1963).

[8] G. A. T. F. da Costa, J. Math. Phys. 38, 1014 (1997).

[9] R. P. Feynman, Statistical Mechanics. A set of lectures. The Benjamin and Cummings Publishing Co., 1972.

[10] H. S. Green and C. A. Hurst, Order-Disorder Phenomena, John Wyley and Sons.

[11] S. G. Brush, Rev. Mod. Phys. 39, 883 (1967).

[12] N. V. Vdovichenko, Soviet Phys. JETP 20, 477 (1965).

[13] Landau and Lifschitz, Statistical Physics, Addison-Wesley, 1969.

[14] M. L. Glasser, Am. J. Phys. 38, 1033, 1970.

[15] C. J. Thompson, Mathematical Statistical Mechanics, Princenton University Press, 1972.

[16] G. F. Newell and E. W. Montroll, Rev. Mod. Phys. 25, 353 (1953).

[17] B. A. Cipra, The American Mathematical Monthly, 94, 937 (1987).

[18] F. Brauer and J. Nohel, The qualitative Theory of ODE, W. A. Benjamin, INC., 1969.

[19] M. Abromowitz and I. A. Stegun, Handbook of Mathematical Functions, Dover, 1972. 